Bull. Austral. Math. Soc.

VoL. 52 (1995) [183-188]

\title{
A NOTE ON RESONANCE PROBLEMS WITH NONLINEARITY BOUNDED IN ONE DIRECTION
}

\author{
To Fu Ma and Luís Sanchez
}

We prove the existence of a solution for a semilinear boundary value problem at resonance in the first eigenvalue. The nonlinearity is assumed to be bounded below or above; no further growth restrictions are assumed.

\section{Statement of the Result}

Many authors have studied the resonance problem

$$
-u^{\prime \prime}-u=g(x, u)+h(x) \text { in }(0, \pi), \quad u(0)=0=u(\pi) .
$$

In order to motivate our analysis we recall that the growth restriction

$$
\limsup _{|u| \rightarrow \infty} \frac{g(x, u)}{u}<3
$$

has been widely used in the literature together with a Landesman-Lazer condition [8] to yield a solution of (1.1): see Ahmad [1], and for conditions of the same type with respect to resonance at higher order eigenvalues, Berestycki and De Figueiredo [2], Iannacci and Nkashama [7] and the references in this article for an account of work in this direction.

Recently $\mathrm{Ha}$ and Song [5] have exploited the boundedness of $g(x, u)$ in one direction, together with a Landesman-Lazer condition, in order to avoid (1.2), allowing the nonlinearity to grow like a power in $u$; we show that indeed that growth restriction may be dropped.

Let us give precise statements of our assumptions and results. Let $g:(0, \pi) \times \mathbb{R} \rightarrow \mathbb{R}$ be an $L^{1}$-Caratheodory function : this means, as usual, that $g$ is measurable in the first variable, continuous in the second one and if $R>0$ is given $\sup _{|u| \leqslant R}|g(x, u)|$ is bounded by some function in $L^{1}(0, \pi)$. Moreover, let us assume that $g$ is bounded below in the sense that for some function $m \in L^{1}(0, \pi)$ we have

$$
|g(x, u)| \leqslant m(x), \quad(x, u) \in(0, \pi) \times(-\infty, 0)
$$

Received 20th October, 1994

This research was supported by JNICT and FEDER under contract STRDA/C/CEN/531/92.

Copyright Clearance Centre, Inc. Serial-fee code: 0004-9729/95 \$A2.00+0.00. 
and

$$
g(x, u) \geqslant-m(x), \quad(x, u) \in(0, \pi) \times(0,+\infty) .
$$

(We need (1.3) because of condition (1.6) to be introduced below. We could symmetrically deal with the case of a Caratheodory function bounded from above with the obvious meaning.) In addition, without loss of generality we take $h$ orthogonal to the first eigenfunction, $\varphi_{1}(x)=\sin x$ :

$$
\int_{0}^{\pi} h(x) \varphi_{1}(x) d x=0
$$

and the corresponding usual Landesman-Lazer condition (which has a meaning because of (1.3)-(1.4)),

$$
\int_{0}^{\pi} g_{-}(x) \varphi_{1}(x) d x<0<\int_{0}^{\pi} g_{+}(x) \varphi_{1}(x) d x
$$

where $g_{-}(x):=\limsup _{u \rightarrow-\infty} g(x, u), g_{+}(x):=\liminf _{u \rightarrow+\infty} g(x, u)$.

Theorem 1. Let $g$ be an $L^{1}$-Caratheodory function and $h \in L^{1}(0, \pi)$ satisfy (1.3), (1.4), (1.5) and (1.6). Then (1.1) has at least one solution.

In section 2 we present the proof of Theorem 1 and make some further comments.

\section{PROOF OF THE THEOREM}

We shall make use of a well-known result from degree theory which we state here in a simple form for the convenience of the reader; we refer for example, to Gaines and Mawhin [6] for a more general version and proof. Let $X$ be a Banach space, $L: D(L) \subset X \rightarrow X$ a linear operator such that $D(L)=\operatorname{Ker}(L) \oplus D_{1}, X=\operatorname{Ker}(L) \oplus X_{1}$ and there exists a continuous partial inverse of $L$, denoted $K: X_{1} \rightarrow D_{1}$. Let $Y$ be another Banach space such that $D(L) \subset Y \subset X$ and the imbedding $D(L) \subset Y$ is compact. Finally, we consider a continuous nonlinear operator $N: Y \rightarrow X$ that takes bounded sets into bounded sets, and the operator equation

$$
L x+N x=0, \quad x \in D(L) .
$$

THEOREM A. Under the above conditions, equation (2.1) has at least one solution provided the following conditions are satisfied: (i) The set of solutions $u$ of the homotopic family of equations

$$
L x+\lambda N x=0, \quad x \in D(L), \quad \lambda \in(0,1)
$$


is bounded in $Y$. (ii) If $P: X \rightarrow \operatorname{Ker}(L)$ denotes the projection along $X_{1}$, then for any open ball $B$ in $\operatorname{Ker}(L)$ with centre at the origin and sufficiently large radius the Brower degree

$$
\operatorname{deg}\left(\left.P N\right|_{\mathrm{Ker}(L)}, B, 0\right)
$$

is defined and is non-zero.

We can now prove Theorem 1 . The operator $L(u)=u^{\prime \prime}+u$ has the above properties with respect to the spaces

$$
X=L^{1}(0, \pi), \quad D(L)=W^{1,2}(0, \pi) \cap W_{0}^{1,1}(0, \pi),
$$

$\operatorname{Ker}(L)=\operatorname{span}\{\sin x\}, X_{1}=\left\{u \in L^{1}(0, \pi): \int_{0}^{\pi} u(x) \sin x d x=0\right\}, D_{1}=X_{1} \cap D(L)$, $Y=C^{1}([0, \pi])$. Accordingly, we consider the family of equations

$$
\begin{cases}u^{\prime \prime}+u+\lambda g(x, u)=\lambda h & x \in(0, \pi) \\ u(0)=0=u(\pi) & 0<\lambda<1,\end{cases}
$$

which is of the type (2.2) if we take $N$ as the Nemytski operator generated by $g(x, u)-$ $h(x)$.

For Theorem A we must check conditions $(i)$ and (ii). Since $\left.P N\right|_{\operatorname{Ker}(L)}$ in this case can be identified with the mapping $\varphi: \mathbb{R} \rightarrow \mathbb{R}$ given by

$$
\varphi(a)=\int_{0}^{\pi} g(x, a \sin x) \sin x d x
$$

and from (1.6) and Fatou's Lemma we obtain

$$
\limsup _{a \rightarrow-\infty} \int_{0}^{\pi} g(x, a \sin x) \sin x d x<0<\liminf _{a \rightarrow+\infty} \int_{0}^{\pi} g(x, a \sin x) \sin x d x
$$

the assertion (ii) readily follows. Therefore it remains to check ( $i$ ). First we note that for any $u$ that solves (2.3) for some $\lambda$ we have, by (1.5)

$$
\int_{0}^{\pi} g(x, u) \sin x d x=0
$$

and, using (1.3)-(1.4) :

$$
\int_{0}^{\pi}|g(x, u)| \sin x d x \leqslant 2 \int_{0}^{\pi} m(x) \sin x d x \equiv C_{1} .
$$

(Hereafter $C_{1}, C_{2}, \ldots$ will denote constants not depending on $u$ or $\lambda$.) 
Now let us decompose $u$ in the form $u(x)=a \sin x+w(x)$ where $a \in \mathbb{R}$ and $w \in D_{1}$. Then $w(x)$ is the only solution in $D_{1}$ of

$$
\begin{gathered}
w^{\prime \prime}+w=-\lambda g(x, u)+\lambda h \\
w(0)=0=w(\pi)
\end{gathered}
$$

and it follows that (denoting by $\|\cdot\|_{C^{1}}$ the norm of $\left.C^{1}([0, \pi])\right)$

$$
\|w\|_{C^{1}} \leqslant \int_{0}^{\pi}|g(x, u)| d x+\int_{0}^{\pi}|h(x)| d x .
$$

Now given $\varepsilon>0$ there exists an integrable positive function $D=D(\varepsilon)>0$ such that

$$
|g(x, u)| \leqslant \varepsilon|u g(x, u)|+D(x)
$$

for all $x \in(0, \pi), u \in \mathbb{R}$. This enables us to estimate the first integral of (2.6):

$$
\begin{aligned}
& \int_{0}^{\pi}|g(x, u)| d x \leqslant \varepsilon \int_{0}^{\pi}|u||g(x, u)| d x+\|D\|_{L^{1}(0, \pi)} \\
& \quad \leqslant \varepsilon|a| \int_{0}^{\pi}|g(x, u)| \sin x d x+\varepsilon \int_{0}^{\pi}|g(x, u)| \sin x \frac{w(x)}{\sin x} d x+\|D\|_{L^{1}(0, \pi)} \\
& \quad \leqslant \varepsilon C_{1}|a|+\varepsilon C_{1} C_{2}\|w\|_{C^{1}}+\|D\|_{L^{1}(0, \pi)}
\end{aligned}
$$

where $C_{2}$ is a constant such that, for all functions $w \in C^{1}([0, \pi])$ such that $w(0)=0=$ $w(\pi)$

$$
\sup _{x \in(0, \pi)}\left|\frac{w(x)}{\sin x}\right| \leqslant C_{2}\|w\|_{C^{1}} .
$$

Combining (2.6), (2.8) and the arbitrariness of $\varepsilon$ we see that, if $u_{n}=a_{n} \sin x+w_{n}(x)$ is a sequence of solutions of (2.3) for corresponding values of $\lambda=\lambda_{n}$ then $u_{n}$ can be unbounded in $C^{1}([0, \pi])$ only if $\left|a_{n}\right| \rightarrow \infty$ (for some subsequence) and then

$$
\frac{w_{n}}{a_{n}} \rightarrow 0 \text { in } C^{1}([0, \pi])
$$

If $a_{n} \rightarrow+\infty$, then $u_{n}(x) \rightarrow+\infty$ for all $x \in(0, \pi)$ and $u_{n}(x)>0$ for all $x \in(0, \pi)$ and $n$ sufficiently large. From (2.3) with $u=u_{n}$ and $\lambda=\lambda_{n}$ we obtain

$$
\int_{0}^{\pi} \liminf g\left(x, u_{n}(x)\right) \sin x d x \leqslant \lim \int_{0}^{\pi} g\left(x, u_{n}(x)\right) \sin x d x=0
$$

which obviously implies a contradiction with the right-hand side of (1.6). If $a_{n} \rightarrow-\infty$ we analogously obtain a contradiction with the left-hand side. Hence $(i)$ is satisfied and the proof is complete. 
Some final remarks are in order. It is apparent that the more regularity $D(L)$ possesses the easier is it to obtain the $C^{1}$-estimate for $w(x)$ (the component of $u(x)$ orthogonal to $\varphi_{1}(x)$ ). In fact this becomes trivial in the following example. Consider the fourth-order one-dimensional boundary value problem

$$
\begin{gathered}
u^{(4)}-u=g(x, u)+h(x) \text { in }(0, \pi) \\
u(0)=u(\pi)=0, \quad u^{\prime \prime}(0)=u^{\prime \prime}(\pi)=0
\end{gathered}
$$

where $g$ and $h$ are as in Theorem 1. Then (2.4) holds and as a straightforward consequence it yields the boundedness of $w$ in $C^{1}[0, \pi]$. To see this, note that for the solution of

$$
u^{(4)}-u=f(x)
$$

with the boundary condition of (2.9) the following estimate holds:

$$
\|w\|_{C^{1}} \leqslant C_{10} \int_{0}^{\pi}|f(x)| \sin x d x .
$$

This follows from the fact that $u$ can be seen as the solution of

$$
\begin{array}{ll}
u^{\prime \prime}-u=U, & U^{\prime \prime}+U=f, \\
u(0)=0=u(\pi), & U(0)=U(\pi)=0 .
\end{array}
$$

If $W$ denotes the component of $U$ orthogonal to $\sin x$, then the properties of the Green's function for the mapping $f \mapsto W$ imply

$$
\|W\|_{C([0, \pi])} \leqslant C_{11} \int_{0}^{\pi}|f(x)| \sin x d x ;
$$

on the other hand it is clear that $\|w\|_{C^{1}} \leqslant C_{12}\|W\|_{C([0, \pi])}$ (this is true even for the $C^{2}$-norm).

As a result, Theorem 1 holds for problem (2.9) (with a very short proof).

Finally, we would like to recall that existence results for the corresponding elliptic problem in higher dimensions can be deduced from the works of Chiappinelli, Mawhin and Nugari [3] and Chiappinelli and De Figueiredo [4]; in particular the result corresponding to Theorem 1 is obtained provided the nonlinearity grows linearly. It is easy to see that our proof applies, under these conditions, in dimensions 2 or 3.

\section{REFERENCES}

[1] S. Ahmad, 'A resonance problem in which the nonlinearity may grow linearly', Proc. Amer. Math. Soc. 92 (1984), 381-384. 
[2] H. Berestycki and D.G. de Figueiredo, 'Double resonance in semilinear elliptic problems', Comm. Partial Differential Equations 6 (1981), 91-120.

[3] R. Chiappinelli, J. Mawhin and R. Nugari, 'Bifurcation from infinity and multiple solutions for some Dirichlet problems with unbounded nonlinearities', Nonlinear Anal. 18 (1992), 1099-1112.

[4] R. Chiappinelli and D.G. De Figueiredo, 'Bifurcation from infinity and multiple solutions for an elliptic system', Differential Integral Equations 6 (1993), 757-771.

[5] Chung-Wei Ha and Wen-Bing Song, 'On a resonance problem with nonlinearities of arbitrary polynomial growth', Bull. Austr. Math. Soc. 48 (1993), 435-440.

[6] R.E. Gaines and J. Mawhin, Coincidence degree and nonlinear differential equations, Lecture Notes in Math. 558 (Springer-Verlag, Berlin, Heidelberg, New York, 1977).

[7] R. Iannacci and M.N. Nkashama, 'Nonlinear boundary value problems at resonance', Nonlinear. Anal. 11 (1987), 455-473.

[8] E.M. Landesman and A.C. Lazer, 'Nonlinear perturbations of a linear elliptic boundary value problem at resonance', J. Math. Mech. 19 (1970), 609-623.

Departamento de Matemática

Universidade Estadual de Maringá

87020-900 Maringá-PR

Brazil

e-mail: matofu@brfuem.bitnet
Departamento de Matemática

Faculdade de Ciências da Universidade de Lisboa

Rua Ernesto de Vasconcelos, Bloco C1

1700 Lisboa

Portugal

e-mail: sanchez@ptmat.lmc.fc.ul.pt 